\title{
MAMÍFEROS DEL BOSQUE MESÓFILO DE MONTAÑA DEL MUNICIPIO DE SAN FELIPE USILA, TUXTEPEC, OAXACA, MEXICO
}

\author{
MARTÍN PÉREZ LUSTRE, RUSBY GUADALUPE CONTRERAS DÍAZ Y \\ ANTONIO SANTOS-MORENO \\ Laboratorio de Ecología Animal. Centro Interdisciplinario de Investigación para el \\ Desarrollo Integral Regional, Unidad Oaxaca, IPN. Hornos 1003, Santa Cruz Xoxocotlán, \\ Oaxaca. C. P. 71230, MÉXICO asantosm90@hotmail.com
}

\begin{abstract}
Resumen: Se realizó el inventario de la mastofauna en el Bosque Mesófilo de Montaña del Municipio de San Felipe Usila en el Distrito de Tuxtepec, Oaxaca, durante cinco periodos mensuales de julio a noviembre de 2005. Se registraron representantes de cinco ordenes, 11 familias, 22 géneros y 23 especies, de las cuales una es endémica, siete están incluidas en alguna categoría de riesgo de acuerdo a normas nacionales e internacionales y cuatro son consideradas indicadoras de buena calidad ambiental, que incluyen tres quirópteros y un carnívoro. Las especies más abundantes fueron el murciélago Sturnira ludovici y el roedor Peromyscus mexicanus.
\end{abstract}

Palabras clave: Riqueza de especies, Bosque Mesófilo de Montaña, San Felipe Usila, Oaxaca, México.

Abstract: A mammal inventory of the cloud forest area of San Felipe Usila town, Tuxtepec, Oaxaca was carried out. The Santa Cruz Tepetotutla and San Antonio del Barrio communities were sampled between July and November 2005. We recorded five orders, 11 families, 22 genera, and 23 species, one species is endemic, seven are considered endangered according to national and international laws, and four are indicators of good quality of the environment, included three bats and one carnivore. The most abundant species was the bat Sturnira ludovici and the rodent Peromyscus mexicanus.

Key words: Species richness, Cloud forest, San Felipe Usila, Oaxaca, Mexico.

\section{INTRODUCCIÓN}

El Bosque Mesófilo de Montaña (BMM) es una comunidad vegetal relictual que, debido a las condiciones particulares de temperatura y humedad que requiere, se encuentra en áreas muy reducidas, por lo que presenta una distribución naturalmente fragmentada. El BMM representa entre 0.5 y $1 \%$ de la superficie total del territorio de México, tiene gran importancia en la diversidad biológica, ya que alberga el $10 \%$ de la 
riqueza florística calculada para todo el país. Por lo tanto este tipo de vegetación es mucho más diverso por unidad de superficie que cualquier otro en el territorio de la república (Rzedowski, 1996). Ocupa el segundo lugar en riqueza de especies de vertebrados, con un $20 \%$ de las especies mesoamericanas endémicas (Flores y Gerez, 1994; Jardel et al., 1993; Luna et al., 1994; Ortega y Castillo, 1996).

En México, Oaxaca es el estado con la mayor superficie de BMM (35,217 ha) en mejor estado de conservación (Ortega y Castillo, 1996; Jardel et al., 1993; WilliamsLinera, 1992), ocupa el primer lugar en cuanto a número de especies de mamíferos terrestres (Ceballos et al., 2005), con 192 (Alfaro et al., 2005), de las cuales 39 son endémicas de la entidad y la mayoría se distribuyen principalmente en BMM y Bosques de Coníferas (Briones-Salas y Sánchez-Cordero, 2004). El BMM es una de las comunidades más amenazadas en Mesoamérica (Ceballos et al., 2005). En los últimos lustros la disminución drástica de la extensión de este tipo de vegetación ha sido causada por la explosión demográfica, la tala clandestina, el cultivo de café, la ganadería y la agricultura de temporal, muchas veces seminómada. En 1971 este bosque ocupaba 1,716,110 ha (0.86 \%) de la superficie del país. Para 1991, sólo se registraron 142,371 ha, que representa el $0.07 \%$ del territorio mexicano. Es decir, en veinte años el área ocupada por esta comunidad vegetal se redujo a menos de una décima parte, a una tasa promedio de 78,687 ha al año (Ortega y Castillo, 1996; Jardel et al., 1993; Williams, 1992). Ante esta grave amenaza, es de gran importancia conocer la diversidad biológica de este ecosistema, por lo que el objetivo de este trabajo es documentar la diversidad de mamíferos en el BMM de San Felipe Usila, en el distrito de Tuxtepec, Oaxaca, México.

\section{MÉTODOS}

\section{Área de Estudio}

San Felipe Usila es un municipio dentro del distrito de Tuxtepec (Figura 1) localizado en la Cuenca del río Papaloapan, al norte del estado y dentro de la provincia fisiográfica de la Sierra Madre Oriental, cuenta con una superficie de $255.17 \mathrm{~km}^{2}$, que representa el 0.267\% en relación al territorio Estatal (Secretaría de Gobernación et al., 1988). Dentro de la zona se presentan los climas: cálido húmedo (Am), semicálido húmedo (ACm) y templado húmedo (Cm) (INEGI, 2004). La mayor parte del territorio de San Felipe Usila está cubierta por bosques y selvas, principalmente Selva Alta Perennifolia, Bosque de Pino, BMM y una porción de terrenos agrícolas (INEGI e INE, 1996). Se realizaron cinco periodos de muestreo comprendidos entre los meses de julio a noviembre de 2005 y se visitaron dos localidades: Santa Cruz Tepetotutla y San Antonio del Barrio. 

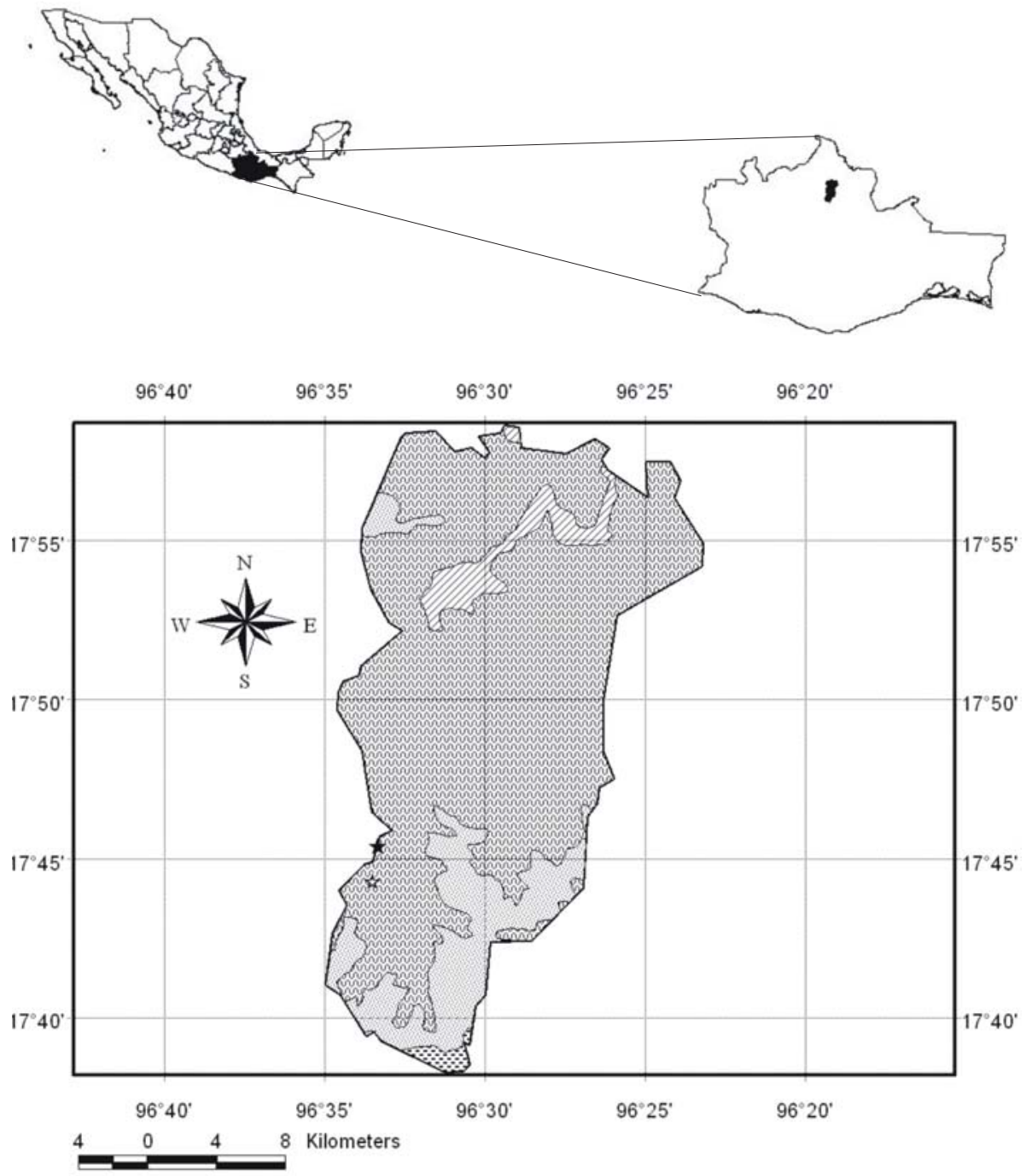

Figura 1. Localización de las localidades de colecta en San Felipe Usila, Tuxtepec, Oaxaca. $\widehat{W}$ Santa Cruz Tepetutla, $\hat{\boldsymbol{~}}$ San Antonio del Barrio. Tipos de vegetación y uso de suelo: $\underset{*}{*}$ Bosque de pino, $\square$ Bosque mesófilo de montaña, $\square$ Manejo agrícola, pecuario y forestal (plantaciones), ${ }_{w}^{W}$ Selva alta perennifolia y subperennifolia, $\square$ Mpio. San Felipe Usila. 


\section{Colecta y Análisis de Datos}

La presencia de especies de mamíferos se documentó a través de muestreo en campo y entrevistas a los habitantes. El registro en campo se realizó a través de métodos directos e indirectos (huellas, excretas, rastros, testimonios y pieles que se pudieran observar). Se establecieron al azar transectos en línea de entre 5 y $10 \mathrm{~km}$ de longitud en cada localidad, los cuales se ubicaron dentro de la vegetación, sobre brechas y veredas.

Para mamíferos de tamaño pequeño se colocaron 50 trampas Sherman cebadas con avena y vainilla, las cuales se colocaron al atardecer y se revisaron a la mañana siguiente; así también, se colocaron trampas durante el día, para incluir dentro del muestreo a las especies de hábitos diurnos durante cinco noches en cada periodo de muestreo, dando un esfuerzo de colecta total de 1250 trampas/noche. Para la captura de murciélagos, se utilizaron dos redes de niebla ( 9 × 2 m; 35 y 60 mm de luz de malla) durante la noche desde las 7 p.m. a las 2 a.m. del día siguiente durante cinco noches en cada periodo de muestreo, lo que representa un esfuerzo de colecta total de $6300 \mathrm{~m}^{2}$ red/hora. La determinación taxonómica de los ejemplares colectados se realizó mediante claves especializadas (Álvarez et al., 1994; Goodwin, 1969; Hall, 1981; Medellín et al., 1997; Reid, 1997).

Se colectaron las excretas encontradas dentro de los transectos, se tomaron impresiones de las huellas encontradas, siguiendo la técnica recomendada por Aranda (2000), la determinación de las especies se realizó por medio de las guías de identificación de rastros de mamíferos de Aranda (2000) y Murie (1982).

Con la finalidad de evaluar si el inventario fue completo se aplicó el modelo asintótico de acumulación de especies de Dependencia Lineal (Soberón y Llorente, 1993). Para el cálculo de los valores de los parámetros del modelo se utilizó el programa Statistica (Statsoft, 2000) y para aleatorizar los datos (100 ocasiones) el programa EstimateS 7.0 (Colwell, 2005).

\section{RESULTADOS Y DISCUSIÓN}

Se registraron un total de cinco órdenes, 11 familias, 22 géneros y 23 especies, representando un $11.98 \%$ de las especies conocidas para el estado de Oaxaca, dentro de los cuales se incluyen tanto mamíferos voladores como no voladores. Para el arreglo taxonómico se siguió a Ramírez-Pulido et al. (2005; Apéndice). El modelo asintótico de Dependencia Lineal muestra que el número de especies registradas en el estudio es una subestimación del número real presente en la zona, ya que la riqueza estimada (Figura 2) es de 26.87 especies (parámetros del modelo: $a=10.191$ y $b=0.379$ ). Es decir, se verificó un 85.18 \% de las especies predichas para la zona y aún restan por registrarse al menos cuatro especies. Para el modelo de Dependencia Lineal se han 
desarrollado estimaciones para estimar el esfuerzo de colecta requerido para alcanzar una proporción de la asíntota (Soberón y Llorente, 1993). Para este estudio se estimarion los periodos de colecta requeridos para alcanzar el 95 \% de la asíntota $\left(t_{q}=0.95\right)$, encontrándose que se requiere tres periodos adicionales de muestreo, siempre y cuando se mantengan constantes el tipo de método de colecta y la tasa de acumulación de registro de especies. Cabe mencionar que los estimadores noparamétricos son más apropiados para sitios en donde no se tiene un inventario completo, como en este caso (Hortal et al., 2006), debido a que las funciones de acumulación son más conservadoras y tienden a subestimar la riqueza de especies presentes en un sitio dado. Por esta razón se estimó el número esperado de especies con el estimador no-paramétrico de Chao, obteniéndose un valor solo ligeramente mayor, de 28 especies (límites del intervalo de 95\% de confianza 24.24 y 43.1).

Las 23 especies registradas o las 27 estimadas con el modelo de Dependencia Lineal representan una riqueza de especies moderada, si se considera por ejemplo, que en el BMM de Omiltemi, Guerrero, se registraron 38 (Jiménez-Almaraz et al., 1993). Una posible explicación de esta diferencia es que de las 54 especies registradas en Omiltemi en los cuatro tipos de vegetación presentes (además del BMM existen bosques de pino, encino y mixto), 17 son murciélagos, y 14 de ellas (25.9\% de la mastofauna) son migratorias.

El número de registros tanto de especies como de individuos pudo haber aumentado con la utilización de otros dispositivos de muestreo como pueden ser las trampas de arpa, que son principalmente útiles para la captura de miembros de la familia Vespertilionidae de tamaño pequeño a mediano (Tidemann y Woodside, 1978) de los cuales no se capturó ninguno en este trabajo, así como para algunos miembros de la familia Molossidae (Francis, 1989) de los que no se cuenta con algún registro. De la misma forma el número de individuos y las relaciones de abundancias pueden incrementarse a través de la colocación de redes verticales y horizontales en el dosel medio (Francis, 1994).

En San Felipe Usila el número de especies de murciélagos (siete) es muy bajo en comparación con la de Omiltemi (17), ninguna de las cuales es considerada como migratoria. En el caso de especies residentes, se observa una notable heterogeneidad en la composición de ambas comunidades, ya que únicamente están presentes en ambas comunidades Anoura geoffoyi y Sturnira ludovici. Este patrón es similar al ya descrito para la flora de este tipo de vegetación a lo largo de toda su distribución en México (Acosta, 2004).

Siete especies de murciélagos y cinco de roedores de talla pequeña registrados en el BMM de San Felipe Usila representan una riqueza similar a la observada en otros estudios de mamíferos en BMM. Por ejemplo Sánchez-Cordero (2001) registró la presencia de seis especies de roedores para la Sierra Mazateca y ocho para la Sierra Mixteca, y dos especies de quirópteros para la Sierra Mazateca y dos para la Mixteca. 


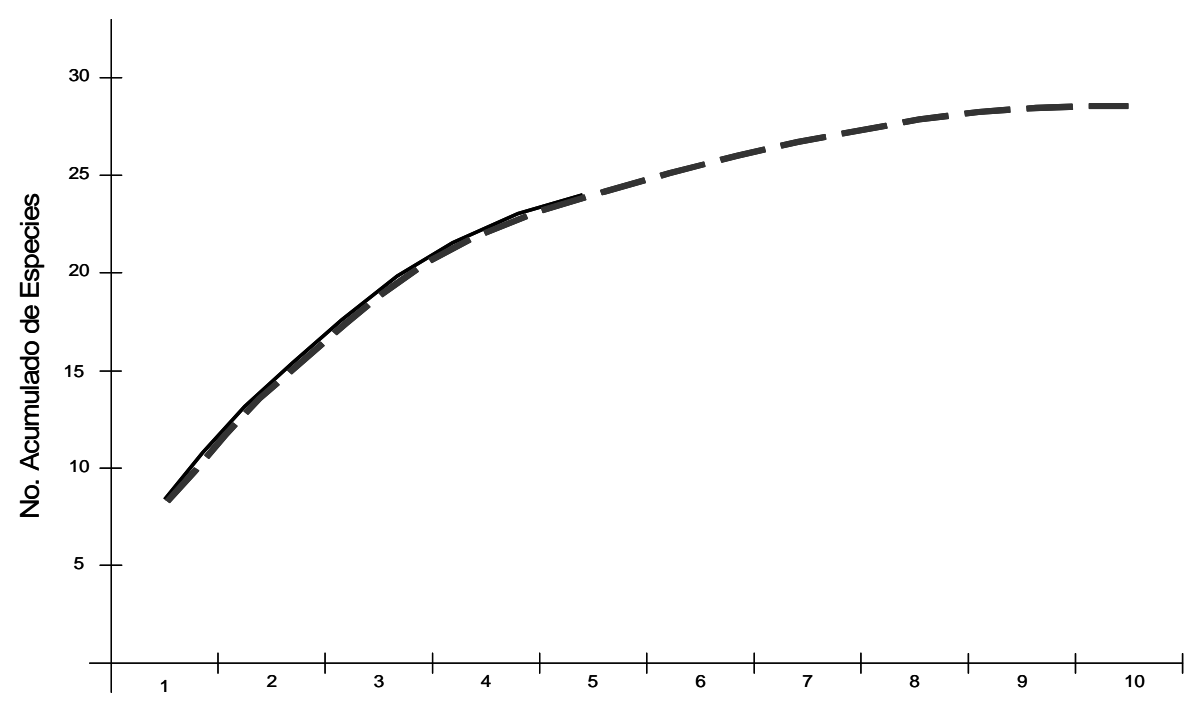

No. Acumulado de Periodos de Colecta

Figura 2. Curva de acumulación aleatorizada observada (—SAcumAleat) y curva de acumulación esperada ( ----- SAcumEsp) de acuerdo al modelo de Dependencia Lineal.

En cuanto a los carnívoros, las cinco especies registradas (Apéndice) en este estudio coinciden con la riqueza de especies conocida para otros sitios. Luna Krauletz (2005) registró dos especies en la porción de BMM del Municipio de Santiago Comaltepec, Oaxaca; en el mismo Municipio Alfaro Espinosa (2006) registró cuatro especies, mientras que Jiménez Almaraz et al. (1993) registraron ocho en Omiltemi, Guerrero.

Finalmente, el buen estado de conservación y la importancia de la mastofauna estudiada se ve reflejada por la presencia de tres especies de murciélagos que pueden ser consideradas como indicadoras de buena calidad ambiental sensu GalindoGonzález, 2004 (Phyllostomus discolor, Carollia sowelli y Sturnira ludovici); la presencia de Panthera onca, que puede considerarse una especie indicadora de buen estado del ecosistema (Aranda, 1994; Miller y Rabinowitz, 2002; Miller et al., 1999); una especie de roedor endémico de México (Oryzomys chapmani) y siete especies que están incluidas en alguna categoría de riesgo de instituciones nacionales o internacionales: CITES, IUCN y/o NOM-059-ECOL-2000 (SEMARNAP, 2002), tres de ellas en peligro de extinción (Panthera onca hernandesii, Leopardus wiedii oaxacensis y Tamandua mexicana mexicana). 


\section{AGRADECIMIENTOS}

A las autoridades y los pobladores del Municipio de San Felipe Usila por las facilidades proporcionadas en el transcurso de esta investigación. El Instituto Politécnico Nacional proporcionó el financiamiento a la Coordinación General de Estudios de Posgrado e Investigación (Proyectos CGEPI-20050200 y CGEPI-20060322) del Instituto Politécnico Nacional. J. L. García García y A. Ma. Alfaro Espinosa revisaron versiones preliminares del documento haciendo valiosas sugerencias.

\section{LITERATURACITADA}

Acosta, S. 2004. Afinidades de la flora genérica de algunos bosques mesófilos de montaña del nordeste, centro y sur de México: un enfoque fenético. Anales del Instituto de Biología, Universidad Nacional Autónoma de México (serie botánica), 75:61-72.

Alfaro Espinosa, A. Ma. 2006. Patrones de diversidad de mamíferos terrestres del municipio Santiago Comaltepec, Oaxaca, México. Tesis de Maestría en Ciencias, Centro Interdisciplinario de Investigación para el Desarrollo Integral Regional, Unidad Oaxaca, Instituto Politécnico Nacional. Oaxaca, México.

Alfaro, A. Ma., J.L. García-García y A. Santos-Moreno. 2005. The false vampire bat Vampyrum spectrum in Oaxaca, México. Bat Research News, 46:145-146.

Álvarez S., T., S. T. Álvarez-Castañeda y J. C. López-Vidal. 1994. Claves para murciélagos mexicanos. Centro de Investigaciones Biológicas del Noroeste S. C. y Escuela Nacional de Ciencias Biológicas (ENCB), IPN, México, D. F.

Aranda, M. 1994. Importancia de los pecaríes (Tayassu spp.) en la alimentación del jaguar (Panthera onca). Acta Zoológica Mexicana (nueva serie), 62:11-22.

Aranda, M. 2000. Huellas y otros rastros de los mamíferos grandes y medianos de México. CONABIO e Instituto de Ecología, A. C., Xalapa, Veracruz, México.

Briones-Salas, M.A. y V. Sánchez-Cordero. 2004. Mamíferos. Pp. 423-447, en: Biodiversidad de Oaxaca (A. J. García-Mendoza, M.J. Ordóñez y M.A. Briones-Salas, eds.). Instituto de Biología-UNAM-Fondo Oaxaqueño para la Conservación de la Naturaleza-World Wildlife Fund, México.

Ceballos, G., J. Arroyo-Cabrales, R.A. Medellín, L. Medrano González y G. Oliva. 2005. Diversidad y conservación de los mamíferos de México. Pp. 21-49, en: Los Mamíferos Silvestres de México (G. Ceballos y G. Oliva, eds.). Fondo de Cultura Económica, México, D. F.

CITES. 2001. Annotated CITES Appendices and Reservations. UNEP-World Conservation Monitoring Centre, Geneve, Switzerland.

Colwell, R.K. 2005. EstimateS: Statistical estimation of species richness and shared species from samples.Version 7.5.User's Guide and application published at: http:// viceroy.eeb.uconn.edu/EstimateS.

Galindo-González, J. 2004. Clasificación de los murciélagos de la región de Los Tuxtlas, Veracruz, respecto a su respuesta a la fragmentación del hábitat. Acta Zoológica Mexicana (nueva serie), 20:239-243. 
Flores, V.O. y P. Gerez. 1994. Biodiversidad y conservación en México: vertebrados, vegetación y uso del suelo. $2^{\mathrm{a}}$ edición. CONABIO-UNAM. México.

Francis, C.M. 1989. A comparison of mist-nets and two designs of harp traps for capturing bats. Journal of Mammalogy, 70:865-870.

Francis, C.M. 1994. Vertical stratification of fruit bats (Pteropodidae) in lowland dipterocarp rainforest in Malaysia. Journal of Tropical Ecology, 10:523-530.

Goodwin, G.G. 1969. Mammals from the state of Oaxaca, Mexico, in the American Museum of Natural History. Bulletin of The American Museum of Natural History, 141:1-269.

Hall, E.R. 1981. The Mammals of North America. Second edition. Vol. 1 y 2. John Wiley y Sons. New York.

Hortal, J., P.A.V. Borges y C. Gaspar. 2006. Evaluating the performance of species richness estimators: sensitivity to sample grain size. Journal of Animal Ecology, 75:274-287.

Instituto Nacional de Estadística, Geografía e Informática (INEGI). 2004. Mapa digital de México. México.

Instituto Nacional de Estadística, Geografía e Informática (INEGI) e Instituto Nacional de Ecología (INE). 1996. Uso de suelo y vegetación. Agrupado por CONABIO. 1998. Escala 1:1,000,000. México.

International Union for Conservation of Nature (IUCN). 2004. 2004 IUCN Red List of Threatened Species. Gland, Suiza.

Jardel, E., A.L. Santiago y M. Muñoz. 1993. El bosque mesófilo de montaña de la Sierra de Manantlán. Tiempos de Ciencia, 30:20-28.

Jiménez-Almaraz, T., J. Juárez Gómez y L. León Paniagua. 1993. Mamíferos, Pp. 503-549, en: Historia natural del parque ecológico estatal Omiltemi, Chilpancingo, Guerrero, México (I. Luna Vega y J. Llorente Bousquets, eds.). Facultad de Ciencias,UNAM y CONABIO. México.

Luna-Krauletz, M.D. 2005. Distribución, abundancia y conservación de carnívoros en Santiago Comaltepec, Sierra Madre de Oaxaca, México. Tesis de Licenciatura. Instituto Tecnológico Agropecuario de Oaxaca, No. 23, Oaxaca, México.

Luna V.I., C.S. Ocegueda y A.O. Ayala. 1994. Florística y notas biogeográficas del bosque mesófilo de montaña de las cañadas de Ocuilan, Estados de Morelos y México. Anales del Instituto de Biología, Universidad Nacional Autónoma de México (serie botánica), 59:6387.

Medellín, R.A., H.T. Arita y O. Sánchez H. 1997. Identificación de los murciélagos de México, clave de campo. Asociación Mexicana de Mastozoología, A. C., México, D. F.

Miller, B. y A. Rabinowitz. 2002. ¿¿Porqué conservar al jaguar? Pp. 303-315, en: El Jaguar en el nuevo milenio. Una evaluación de su estado, detección de prioridades y recomendaciones para la conservación de los jaguares en América (R. A. Medellín, C. Chetkiewicz, A. Rabinowitz, K.H. Redford, J.G. Robinson, E. Sanderson y A. Taber, eds.). Universidad Nacional Autónoma de México / Wildlife Conservation Society, México D. F.

Miller, B., R. Reading, J. Strittholt, C. Carroll, R. Noss, M. Soulé, O. Sánchez, J. Terborg, D. Brightsmith, T. Cheeseman y D. Foreman. 1999. Using focal species in the design of nature reserve networks. Wild Earth, 11:81-92.

Murie, O.J. 1982. A field guide to animal tracks. Segunda edición. Houghton Mifflin Company, Boston, New York. 
Ortega, E.S. y G.C. Castillo. 1996. El bosque mesófilo de montaña y su importancia forestal. Ciencias, 43:32-39.

Ramírez-Pulido, J., J. Arroyo-Cabrales y A. Castro-Campillo. 2005. Estado actual y relación nomenclatural de los mamíferos terrestres de México. Acta Zoológica Mexicana (nueva serie), 21:21-82.

Reid, F.A. 1997. A field guide to the mammals of Central America and southeast Mexico. Oxford University Press, New York.

Rzedowski, J. 1996. Análisis preliminar de la flora vascular de los bosques mesófilos de montaña de México. Acta Botánica Mexicana, 35:25-44.

Sánchez-Cordero, V. 2001. Elevation gradients of diversity for rodents and bats in Oaxaca, Mexico. Global Ecology and Biogeography, 10:63-76.

Secretaría de Medio Ambiente, Recursos Naturales y Pesca (SEMARNAP). 2002. Norma Oficial Mexicana NOM-059-ECOL-2000. Protección ambiental, especies de flora y fauna silvestres de México, categorías de riesgo y especificaciones para su inclusión, exclusión o cambio, y lista de especies en riesgo. Diario Oficial de la Federación, 1:1-62.

Secretaría de Gobernación, Centro Nacional de Estudios Municipales, Gobierno del Estado de Oaxaca y Los Municipios de Oaxaca. 1988. Enciclopedia de los Municipios de México. Talleres Gráficos de la Nación. México, D. F. en: http://www.e-local.gob.mx/work/ templates/enciclo/oaxaca/municipios/20136a.htm

Soberón M., J. y J. Llorente B. 1993. The use of species accumulation functions for the prediction of species richness. Conservation Biology, 7:480-488.

Statsoft, Inc. 2000. Statistica for Windows. Tulsa, Oklahoma, USA.

Tidemann, C.R. y D.P. Woodside. 1978. A collapsible bat trap and comparison of results obtained with the trap and with mist-nets. Australian Wildlife Research, 5:355-362.

Williams-Linera, G. 1992. Ecología del paisaje y el bosque mesófilo en el centro de Veracruz. Ciencia y Desarrollo, 105:133-138. 

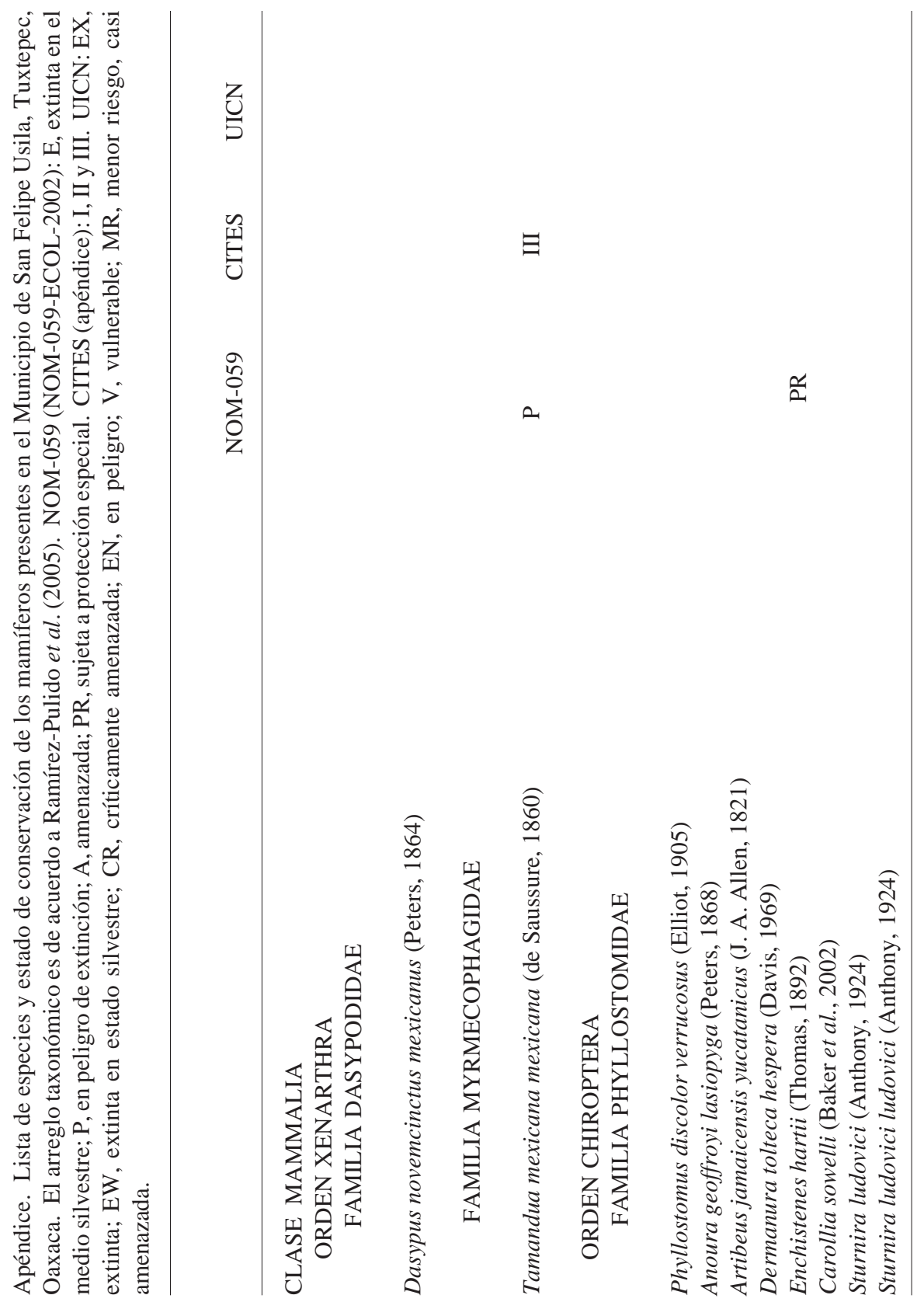


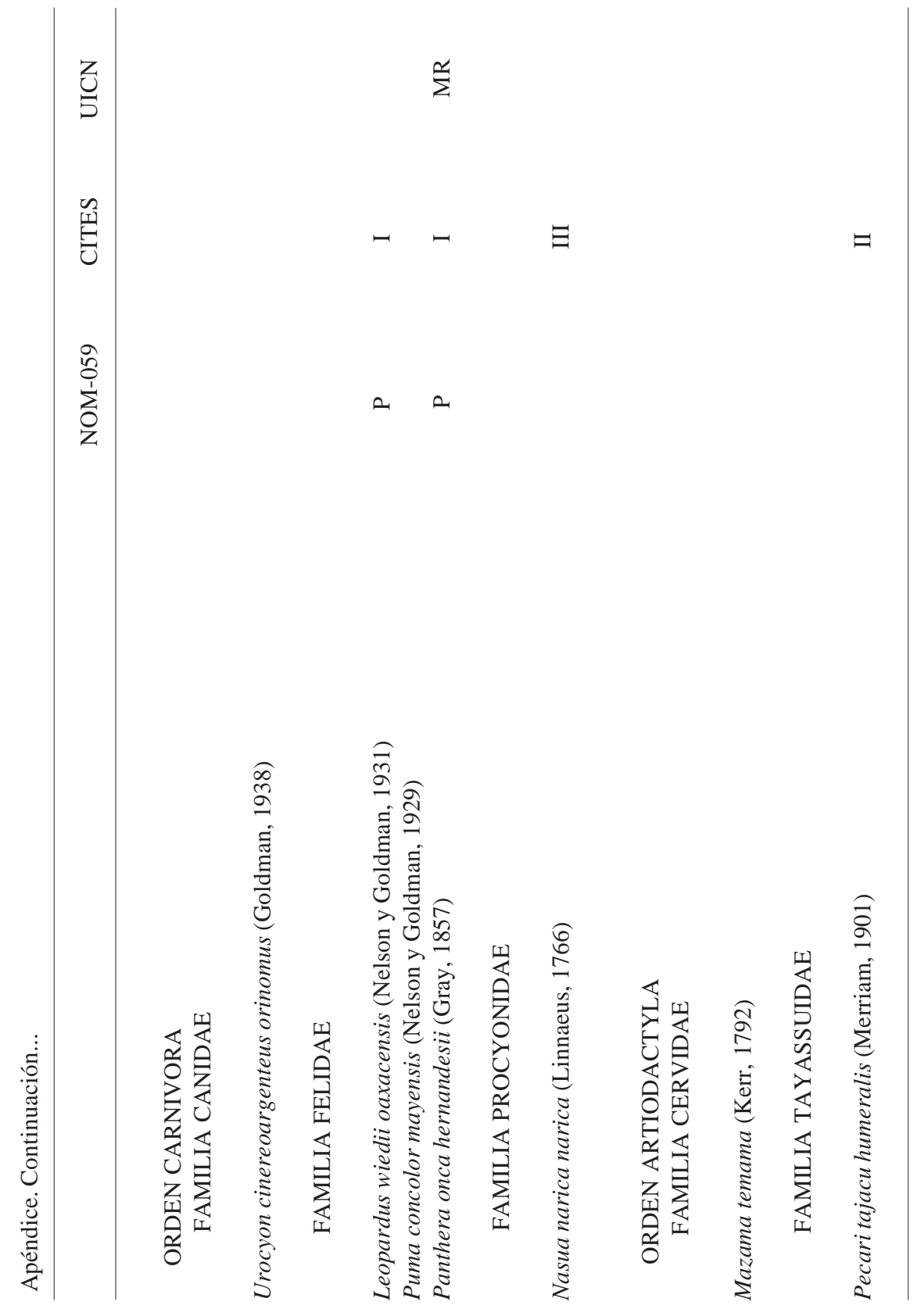


vol. 10

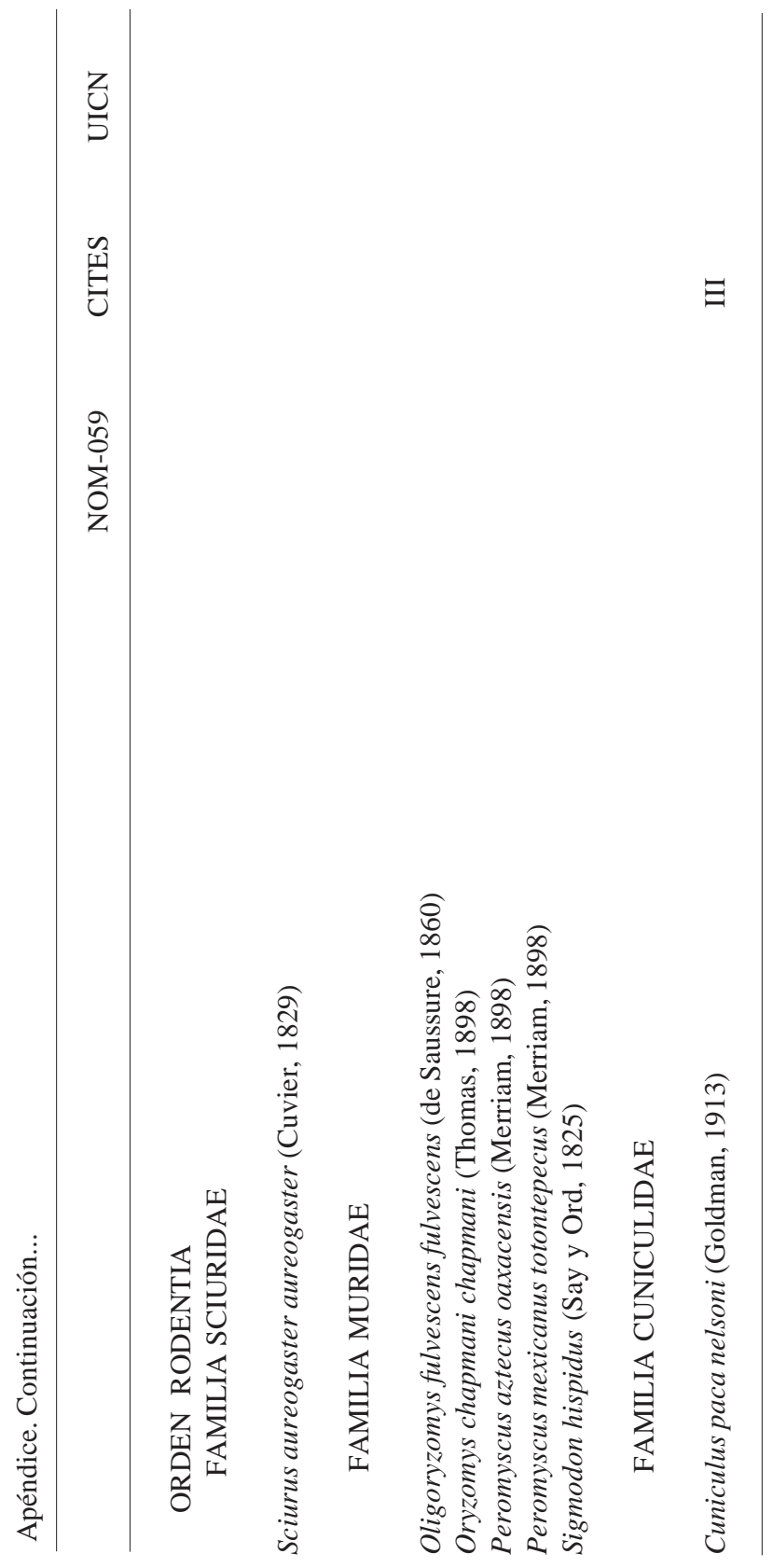

